EPJ Web of Conferences 66, 02019 (2014)

DOI: $10.1051 /$ epjconf/ 20146602019

(C) Owned by the authors, published by EDP Sciences, 2014

\title{
Ground-state configuration of neutron-rich Aluminum isotopes through Coulomb Breakup
}

S. Chakraborty ${ }^{1}$, U. Datta Pramanik ${ }^{1,2, a}$, T. Aumann ${ }^{3,2}$, S. Beceiro ${ }^{4}$, K. Boretzky ${ }^{2}$, C. Caesar ${ }^{2}$, B.V. Carlson ${ }^{5}$, W. N. Catford ${ }^{6}$, S.Chatterjee ${ }^{1}$, M. Chartier ${ }^{7}$, D. Cortina-Gil ${ }^{4}$, G.De. Angelis ${ }^{8}$, D. Gonzalez-Diaz ${ }^{2}$, H. Emling ${ }^{2}$, P. Diaz Fernandez ${ }^{4}$, L. M. Fraile ${ }^{9}$, O. Ershova ${ }^{2}$, H. Geissel ${ }^{2,10}$, M. Heil $^{2}$, B. Jonson ${ }^{11}$, A. Kelic ${ }^{2}$, H. Johansson ${ }^{11}$, R. Kruecken ${ }^{12}$, T. Kroll ${ }^{3}$, J. Kurcewicz ${ }^{2}$, C. Langer ${ }^{2}$, T. Le Bleis ${ }^{12}$, Y. Leifels ${ }^{2}$, G. Munzenberg ${ }^{2}$, J. Marganiec ${ }^{2}$, C. Nociforo ${ }^{2}$, A. Najafi ${ }^{13}$, V. Panin ${ }^{2}$, S. Paschalis ${ }^{3}$, S. Pietri², R. Plag², A. Rahaman', R. Reifarth², V. Ricciardi², D. Rossi², J. Ray ${ }^{1}$, H. Simon ${ }^{2}$, C. Scheidenberger ${ }^{2,10}$, S. Typel ${ }^{2}$, J. Taylor ${ }^{7}$, Y. Togano ${ }^{15}$, V. Volkov ${ }^{3}$, H. Weick ${ }^{2}$, A.Wagner ${ }^{15}$, F. Wamers ${ }^{2}$, M. Weigand ${ }^{2}$, J.S. Winfield ${ }^{2}$, D. Yakorev ${ }^{15}$, and M. Zoric ${ }^{2}$ for s306 collaboration

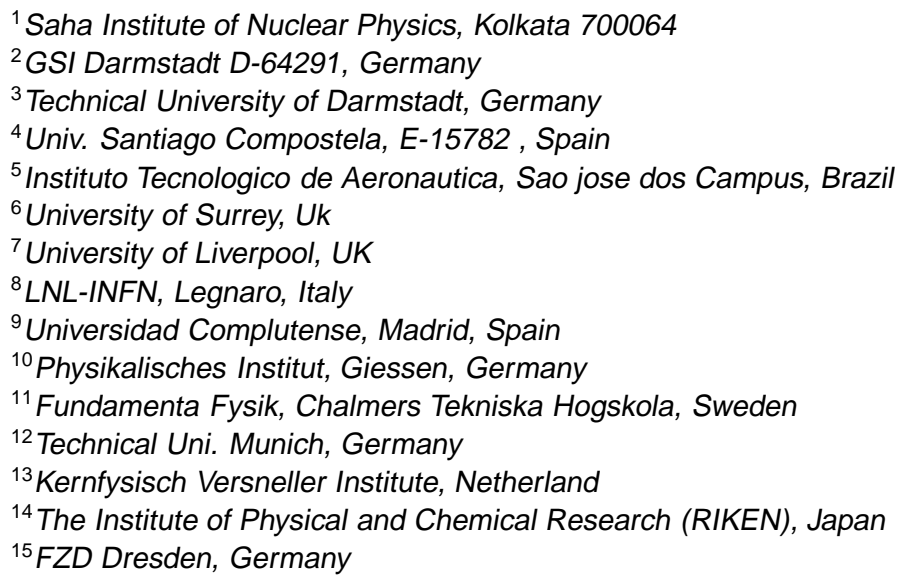

\begin{abstract}
Neutron-rich ${ }^{34,35} \mathrm{Al}$ isotopes have been studied through Coulomb excitation using LAND-FRS setup at GSI, Darmstadt. The method of invariant mass analysis has been used to reconstruct the excitation energy of the nucleus prior to decay. Comparison of experimental CD cross-section with direct breakup model calculation with neutron in $p_{3 / 2}$ orbital favours ${ }^{34} \mathrm{Al}(\mathrm{g} . s) \otimes v_{p_{3 / 2}}$ as ground state configuration of ${ }^{35} \mathrm{Al}$. But ground state configuration of ${ }^{34} \mathrm{Al}$ is complicated as evident from $\gamma$-ray spectra of ${ }^{33} \mathrm{Al}$ after Coulomb breakup of ${ }^{34} \mathrm{Al}$.
\end{abstract}

\footnotetext{
aPresented at INPC2013; corresponding author: ushasi.dattapramanik@saha.ac.in
} 


\section{Introduction}

The region of the nuclear chart around neutron magic number, $\mathrm{N} \sim 20$ and proton number $(\mathrm{Z}), 10 \leq \mathrm{Z} \leq$ 12 is known as the "Island of Inversion". One of the interesting exotic nuclear phenomena observed in the "Island of Inversion" nuclei is breakdown of "magic numbers". The valence neutron(s) of these nuclei, even in their ground state, are most likely occupying the upper $p f$ orbitals which are normally lying above $s d$ orbitals, $\mathrm{N} \sim 20$ shell closure. It was first hinted by Thibault et al. [1] in mid 1970's through mass measurement of sodium isotopes ( $\left.{ }^{31,32} \mathrm{Na}\right)$. Later, Motobayashi et al. [2] measured enhanced B(E2) value of first excited state of ${ }^{32} \mathrm{Mg}$ which was explained considering intruder orbitals contribution in the ground state configuration. The $\gamma$-ray spectroscopy after Coulomb breakup is a direct probe for studying ground state configuration of loosely bound nuclei [3]. Hence, study of the nuclei in and around 'Island of Inversion' through invariant mass analysis with $\gamma$-ray energy tagging (for separating ground state from excited state of the core after Coulomb breakup) can provide detailed ground state information $[3,5]$. Further, Coulomb breakup data can provide radiative capture cross section which may be useful in Astrophysical scenario [6]. Nuclei like ${ }^{34,35} \mathrm{Al}$ are lying at the boundary of this 'Island of Inversion'. Little experimental information about their ground state configurations are available in literature [7-9]. g-factor measurement of ${ }^{34} \mathrm{Al}$ by Himpe et al. [7] showed the need of intruder components in ${ }^{34} \mathrm{Al}$ wave function to account for the observed large magnetic moment. Recently, an experiment (S306) has been performed [5] at GSI, Darmstadt using LAND-FRS setup [10] to explore the neutron-rich exotic nuclei in and around N 20 'Island of Inversion' (Fig. 1(b) ).

\section{Experiment and method of analysis}

A ${ }^{40} \mathrm{Ar}$ primary beam at $530 \mathrm{MeV} / \mathrm{u}$ was delivered by the SIS facility at GSI, Darmstadt. Short-lived radioactive nuclei were produced by the fragmentation of the primary beam $\left({ }^{40} \mathrm{Ar}\right.$ ) on a ${ }^{9} \mathrm{Be}$ production target $\left(8 \mathrm{gm} / \mathrm{cm}^{2}\right)$. The secondary beams were separated according to the magnetic rigidities by the fragment separator (FRS). A suitable degrader was used and the resulting beam contained isotopes
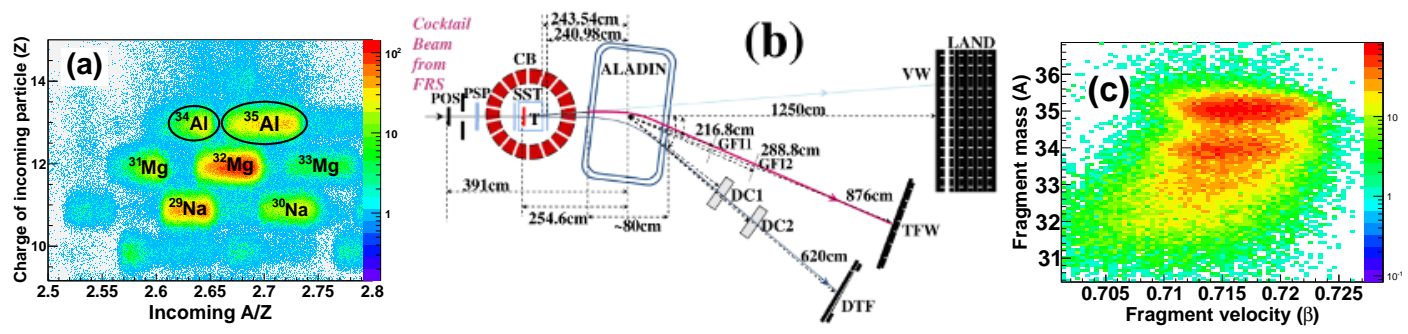

Figure 1. (a). Plot for In-coming beam Identification. (b). Schematic diagram of experimental setup. (c). Plot of outgoing Fragment mass of $\mathrm{Al}$ isotopes $\mathrm{Vs}$ fragment velocity.

$\left({ }^{29,30} \mathrm{Na},{ }^{31-33} \mathrm{Mg},{ }^{34,35} \mathrm{Al}\right.$ etc.) with similar mass to charge ratio (A/Z between 2.55 to 2.85 ) as shown in Fig. 1(a). Unique identification of the secondary beam was done by energy loss measurement in Position Sensitive silicon Pin diode (PSP) and time of flight measurements between two scintillator detectors (S8 and POS) before the reaction target. The secondary cocktail beam was bombarded on different reaction targets. Thick lead $\left(2 \mathrm{gm} / \mathrm{cm}^{2}\right)$ as reaction target was used for studying electromagnetic excitation of the projectiles and $\mathrm{C}\left(935 \mathrm{mg} / \mathrm{cm}^{2}\right)$ as reaction target was used for measuring nuclear contribution. In order to detect the $\gamma$-rays from the excited projectile or projectile like fragments, the reaction target was surrounded by the $4 \pi$-crystal ball spectrometer which consists of 162 $\mathrm{NaI}$ crystals. Eight double-sided Silicon $\mu$-Strip Trackers (SSTs) were placed enclosing the reaction 
INPC 2013

Table 1. Coulomb dissociation cross-section of ${ }^{35} \mathrm{Al}$ nuclei up to $10 \mathrm{MeV}$ excitation energy

\begin{tabular}{cccccc}
\hline Isotope & $\begin{array}{c}\text { ground state } \\
\text { spin [13] } \\
\text { and parity }\end{array}$ & $\begin{array}{c}S_{n} \\
(\mathrm{MeV})\end{array}$ & & Coulomb dissociation (CD) Cross-section (mb) & \\
& & & $\begin{array}{c}\text { neutron } \\
\text { orbital }\end{array}$ & $\begin{array}{c}\text { Direct } \\
\text { Beakup model }\end{array}$ & Experiment \\
& & & & 69 & $65 \pm 10$ \\
${ }^{35} \mathrm{Al}$ & $\left(5 / 2^{+}\right)$ & 5.2 & $p_{3 / 2}$ & 3 & \\
& & & $f_{7 / 2}$ & &
\end{tabular}

target in $4 \pi$ solid angle. After reaction at the secondary target, reaction fragments and particles like proton(s), alpha(s) and neutron(s) from the decaying fragments, are forward focused due to Lorentz boost and passes through A Large Dipole Magnet (ALADIN). Reaction fragments, deflected by ALADIN according to their A/Z ratios, were tracked by two large scintillating fiber detectors (GFIs) and detected by the time of flight wall (TFW) [11]. Lighter protons were deflected at higher angles (w.r.t to heavier fragments) inside ALADIN. These protons were tracked via two Drift chambers (DCs) and detected by the Proton Wall (DTF) [11]. The trajectories of neutrons remain unchanged and were detected by the Large Area Neutron Detector (LAND) [11]. Data analysis has been performed using CERN-ROOT platform and program developed at GSI, Darmstadt and SINP, Kolkata [12].

The measured position resolution for s- and k-side of the silicon strip trackers placed before ALADIN were $135.5 \pm 0.5 \mu \mathrm{m}$ and $159.1 \pm 0.6 \mu \mathrm{m}$, respectively. The measured position resolution $\left(\sigma_{x}\right)$ of the other tracking detector GFI was $3.18 \pm 0.14 \mathrm{~mm}$. The calibration of TFW, DTF and the neutron detector (LAND) has been presented in Ref. [11]. Mass to charge ratio of the reaction fragments were identified by reconstructing the magnetic rigidities inside ALADIN and velocity measurements of the fragments. Fig. 1(c) shows a 2D-plot of fragment mass for Aluminum isotopes against their velocities. All the $\gamma$-rays detected by the $4-\pi$ crystal ball detector in the laboratory frame were subjected
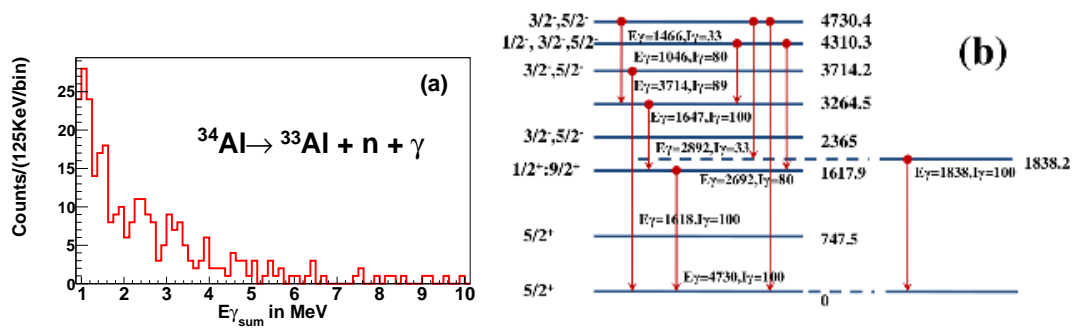

Figure 2. (a). $\gamma$-sum spectra of ${ }^{33} \mathrm{Al}$ obtained after Coulomb breakup of ${ }^{34} \mathrm{Al}$ using lead target after Doppler correction. (b) Level scheme of ${ }^{33} \mathrm{Al}$ from literature.

to Doppler correction resulting in the reconstructed energy in the rest frame. ${ }^{33} \mathrm{Al}$ reaction fragments were obtained after Coulomb break-up of ${ }^{34} \mathrm{Al}$. Fig. 2 (a) show the $\gamma$-sum spectrum of ${ }^{33} \mathrm{Al}$ obtained in coincidence of the fragment with the one neutron after Doppler correction. By measuring the fourmomenta $\mathrm{P}_{i}$ of all the decay products, the excitation energy $\mathrm{E}^{*}$ of the nucleus prior to decay can be reconstructed on an event-by-event basis by analyzing the invariant mass $\mathrm{M}$. If E $\gamma$ be the energy of the released $\gamma$-rays then for one neutron removal channel the excitation energy is given by

$$
E^{*}=\left[\left(m_{n} c^{2}\right)^{2}+\left(m_{f} c^{2}\right)^{2}+2 \gamma_{n} \gamma_{f}\left(m_{n} c^{2}\right)\left(m_{f} c^{2}\right)\left(1-\beta_{n} \beta_{f} \cos \theta_{n f}\right)\right]^{1 / 2}-m_{p} c^{2}+E_{\gamma}
$$


$m_{n(f)}, \beta_{n(f)}$ and $\gamma_{n(f)}$ denote the ground state rest mass, the velocity $(v / c)$ and Lorentz factor of neutron(fragment), respectively. $m_{p}$ denotes the projectile rest mass. $\theta_{n(f)}$ represents the relative angle between the neutron and fragment in the laboratory frame. The relative kinetic energy between the neutron and fragment is $E_{r e l}=E^{*}-S_{n}$ where, $S_{n}$ is one neutron separation energy and $\mathrm{S}_{n}$ for ${ }^{34} \mathrm{Al}$ and ${ }^{35} \mathrm{Al}$, is 2.67 and $5.22 \mathrm{MeV}$, respectively.

\section{Results and Discussion}

The ground state configuration of ${ }^{34} \mathrm{Al}(\mathrm{N}=21)$ isotope is more complicated as it is evident from observed $\gamma$-ray spectra of ${ }^{33} \mathrm{Al}$ obtained after Coulomb breakup of ${ }^{34} \mathrm{Al}$. On the other hand small contribution of characteristic $\gamma$-rays has been observed corresponding to ${ }^{34} \mathrm{Al}$ after Coulomb breakup of ${ }^{35} \mathrm{Al}$. Table I shows experimental and calculated Coulomb dissociation cross-section of ${ }^{35} \mathrm{Al}(\mathrm{N}=22)$ up to excitation energy $10 \mathrm{MeV}$. Comparison of experimental CD cross-section with direct breakup model calculation favours ${ }^{34} \mathrm{Al}(\mathrm{g} . s) \otimes v_{p_{3 / 2}}$ as ground state configuration of ${ }^{35} \mathrm{Al}$. Monte-Carlo shell model calculation by Otsuka et. al. [14] has predicted the lowering of $2 \mathrm{p}_{3 / 2}$ orbital for aluminium isotopes around the $\mathrm{N} \sim 20$ shell gap. However, using similar technique it has been observed that the valence neutron of the ground state of neutron-rich $\mathrm{Na}$ isotopes $(\mathrm{N}=18,19)$ occupy $s_{1 / 2}$ orbital [11].

\section{Acknowledgements}

The authors are thankful to the accelerator people of GSI, for their support during the experiment. Author, U. Datta Pramanik gratefully acknowledges the Alexander von Humboldt (AvH) foundation for the support during proposing, planning and performing the experiment at GSI, Darmstadt. This work has been partially funded by the XIth plan, SEND project (PIN:11-R\&D-SIN-5.11-0400), DAE, Govt. of India.

\section{References}

[1] C. Thibault et al., Phys. Rev. C 12, 644 (1975)

[2] T. Motobayashi et al., Physics Lett. B 346, 9 (1995)

[3] U. Datta Pramanik et al., Phys. Lett. B 551, 63 (2003)

[4] T. Nakamura et al., Phys. Rev. Lett. 83, 1112 (1999)

[5] U. Datta Pramanik et al., A proposal for experiment S306 (2005)

[6] U. Datta Pramanik, Prog. Part. Nucl. Phys. 59, 183 (2007).

[7] P. Himpe et al., Phys. Lett. B 643, 257 (2006)

[8] K. Shimada et al., Physics Lett. B 714, 246 (2012)

[9] C. Nociforo et al., Phys. Rev. C 85, 044312 (2012)

[10] http://www-land.gsi.de/a_new_land/index.html

[11] A. Rahaman et al. , Eur. Phys. J. (INPC2013)

[12] U. Datta Pramanik et al., (to be published)

[13] http://www.nndc.bnl.gov

[14] T. Otsuka et al. ; Phys. Rev. Lett. 104, 012501 (2010) 